\title{
Erratum to: A semiclassical treatment of the interaction of non-uniform electric fields with the electric quadrupole moment of a neutral particle
}

\section{K. Bakke ${ }^{\mathrm{a}}$}

Departamento de Física, Universidade Federal da Paraíba, Caixa Postal 5008, 58051-900, João Pessoa, PB, Brazil

Original article: Eur. Phys. J. Plus (2019) 134: 76, https://doi.org/10.1140/epjp/i2019-12489-1

Received: 22 July 2019

Published online: 13 September 2019

(C) Società Italiana di Fisica / Springer-Verlag GmbH Germany, part of Springer Nature, 2019

After publication, the author noticed that some equations needed amendments. Here follows their correct version:

1) Equation (15) should read

$$
\begin{aligned}
\frac{1}{\hbar} \int_{0}^{r_{2}} q(r) \mathrm{d} r & =\frac{1}{\hbar} \sqrt{2 m \mu Q} \int_{0}^{r_{2}} \sqrt{r_{2}-r} \mathrm{~d} r \\
& =\frac{2}{3 \hbar} \sqrt{2 m \mu Q}\left(r_{2}\right)^{3 / 2} .
\end{aligned}
$$

2) Equation(16) should read

$$
\mathcal{E}_{n, 0}=\mu Q\left[\frac{9 \hbar^{2} \pi^{2}}{8 m \mu Q}\left(n-\frac{1}{2}\right)^{2}\right]^{1 / 3}
$$

3) Equation (29) should read

$$
\begin{aligned}
\frac{1}{\hbar} \int_{0}^{r_{2}} q(r) \mathrm{d} r & =\frac{r_{2}}{\hbar} \sqrt{m Q E_{0}} \int_{0}^{\infty} \sqrt{x} e^{-x} \mathrm{~d} x \\
& =\frac{r_{2}}{\hbar} \sqrt{m Q E_{0}} \frac{\sqrt{\pi}}{2} .
\end{aligned}
$$

4) Equation (30) should read

$$
\mathcal{E}_{n, 0}=\frac{Q E_{0}}{2} \ln \left(\frac{\hbar}{r_{0}} \sqrt{\frac{\pi}{Q E_{0} m}}\left[n-\frac{1}{2}\right]\right)-\frac{Q E_{0}}{4} .
$$

These corrections do not affect the physical conclusion of the paper.

${ }^{a}$ e-mail: kbakke@fisica.ufpb.br 